\title{
Empreintes et emprunts des avant-gardes dans la poésie espagnole contemporaine : la caja de prosa dans Maniluvios (1972), de José-Miguel Ullán
}

\section{Raquel Lledós}

\section{(2) OpenEdition}

\section{Journals}

Édition électronique

URL : https://journals.openedition.org/cher/11614

DOI : $10.4000 /$ cher. 11614

ISSN : 2803-5992

\section{Éditeur}

Presses universitaires de Strasbourg

\section{Édition imprimée}

Date de publication : 1 décembre 2012

Pagination : 157-175

ISBN : 978-2-35410-049-0

ISSN : 1968-035X

\section{Référence électronique}

Raquel Lledós, «Empreintes et emprunts des avant-gardes dans la poésie espagnole contemporaine : la caja de prosa dans Maniluvios (1972), de José-Miguel Ullán », reCHERches [En ligne], 9 | 2012, mis en ligne le 14 février 2022, consulté le 17 février 2022. URL : http://journals.openedition.org/cher/11614 ; DOI : https://doi.org/10.4000/cher.11614

\section{(c)}

Ce(tte) œuvre est mise à disposition selon les termes de la Licence Creative Commons Attribution -

Pas d'Utilisation Commerciale - Partage dans les Mêmes Conditions 4.0 International. 


\title{
Empreintes et emprunts des avant-gardes dans la poésie espagnole contemporaine: la caja de prosa dans Maniluvios (1972), de José-Miguel Ullán
}

\author{
Raquel Lledós \\ Université de Franche-Comté
}

\begin{abstract}
T a poésie de José-Miguel Ullán, néà Salamanque en 1944, est profondément Lempreinte de la lettre et de l'esprit des avant-gardes'. Poète mais aussi artiste-peintre, commissaire d'exposition et critique d'art contemporain, son œuvre littéraire se caractérise par un échange permanent avec les arts plastiques. La série Funeral mal, publiée à Paris entre 1972 et 1985 dans les éditions de la fondation Maeght, est composée de six livres réalisés en collaboration avec les artistes Eduardo Chillida, Pablo Palazuelo, Vicente Rojo, Antonio Saura, Antoni Tàpies et Joan Miró. La vocation expérimentale, ainsi que l'exploration des limites entre les genres littéraires et entre les disciplines artistiques, rendent sa production poétique très
\end{abstract}

1 José-Miguel Ullán avait réussi à rassembler, un an avant son décès en mai 2009, l'ensemble de son œuvre poétique dans un volume d'environ mille pages: Ondulaciones. Poesía reunida (1968-2007), Galaxia Gutemberg, 2008. Tout comme son anthologie poétique précédente, Ardicia (Antología poética, 1964-1994), publiée en 1994 dans la collection Cátedra Letras hispánicas, Ondulaciones était précédée d’un excellent prologue de Miguel Casado. Dans Ardicia, une brève note biographique avait aussi été incluse: «José-Miguel Ullán nace en Villarino de los Aires (Salamanca) el 30 de octubre de 1944. Tras estudiar el bachillerato en Salamanca, en 1959 se traslada a Madrid, donde inicia estudios de Ciencias políticas, Ciencias sociales y Filosofía y Letras. Vive en París entre 1966 y 1976. École Pratique des Hautes Études: cursos con Jean Vilar, Roland Barthes y Lucien Goldmann. Trabaja en la ORTF. De vuelta a Madrid, desarrolla una intensa actividad periodística; de ella se podría destacar el programa de TVE Tatuaje y la creación en Diario 16 del suplemento "Culturas". Actualmente colabora en El País». Dans les années 90, José-Miguel Ullán fonda et dirigea la maison d'édition Ave del Paraíso. 
proche du projet créatif mis en œuvre par les avant-gardes dans la première moitié $\mathrm{du} \mathrm{xx}^{\mathrm{e}}$ siècle. Les traces de cet héritage se révèlent par le recours à des pratiques comme le collage, le simultanéisme ou l'objet trouvé, tout comme par l'exploitation de la dimension visuelle de l'écriture ou par le rapprochement entre la littérature et les arts. À l'instar de mouvements tels que le futurisme, le dadaïsme ou le surréalisme, l'œuvre poétique de JoséMiguel Ullán défie les conventions littéraires et bouscule les habitudes du lecteur, explore des pratiques de création et des expériences de réception nouvelles, tout en proposant une réflexion critique sur la représentation artistique du monde.

Malgré l'extrême diversité des manifestations artistiques et littéraires d'avant-garde, il est possible d'identifier des tendances esthétiques partagées par les différents mouvements, ainsi que d'établir une poétique commune permettant d'intégrer des pratiques de création plurielles, souvent déterminées par les spécificités propres à chaque discipline artistique. Déjà en 1925, Guillermo de Torre, l'auteur de Literaturas europeas de vanguardia, avait souligné le caractère international de l'esthétique des avant-gardes, en faisant remarquer «la extensión ecuménica de su espíritu y de ciertas normas» (1971: 26). Dans l'ouvrage Les avant-gardes littéraires au $\mathrm{XX}^{e}$ siècle, paru en 1986, Jean Weisgerber parvient à identifier une série d'attitudes positives et négatives qui marquent l'ensemble des structures artistiques d'avant-garde: l'esprit antitraditionnel, la révolte, la rupture et le renversement, l'éloge de la nouveauté, l'obsession du futur, la liberté, l'expérimentation, l'esprit ludique. Pour Weisgerber,

les tendances esthétiques des avant-gardes naissent et se développent sous le signe d'une négation radicale: le refus catégorique et global de l'art et de la littérature antérieurs (1986: 633)2.

Plus récemment, en 1998, Ana María Gómez Torres, dans son livre La retórica de la nada: en torno a la poética de las vanguardias, définit la poétique des avant-gardes comme un bouleversement radical des catégories

2 L'ampleur de ce rejet n'atteint pas seulement l'héritage transmis par la voie de la tradition, mais aussi les mouvements d'avant-garde précédents: «Ces renversements de positions relèvent d'ailleurs de la plus pure stratégie d'avant-garde: le dernier mouvement, en s'insurgeant, fait table rase de toutes les avant-gardes antérieures, au nom des mêmes principes» (Weisgerber, 1986: 634). 
fondamentales de l'esthétique occidentale, de la création à la lecture, en passant par la représentation ou mimésis ${ }^{3}$.

Notre propos est d'interroger la relation entre la poésie de José-Miguel Ullán et les avant-gardes en nous centrant sur l'analyse des cajas de prosa dans Maniluvios (1972). À partir de l'étude des citations intertextuelles et des références intergénériques, nous tenterons de montrer dans quelle mesure son œuvre partage des aspects essentiels de la poétique des avantgardes: l'abolition des frontières entre prose et poésie, la fusion entre théorie et pratique, ainsi que le franchissement des limites entre la littérature et les arts.

\section{De la théorie à la poésie: le manifeste}

Dans leur volonté de s'affranchir des normes et des conventions qui président aux pratiques artistiques, les artistes d'avant-garde vont même tenter d'abolir la séparation entre théorie et création. Le manifeste, genre par excellence du discours réflexif et rhétorique, va s'inspirer des découvertes formelles mises en place par les poètes dans leur production littéraire. Ainsi, lorsque Guillaume Apollinaire compose en 1913 son manifeste L'Antitradition futuriste, il n'hésite pas à mêler des typographies différentes, à jouer avec la disposition des blancs sur l'espace. Ce travail sur le signifiant graphique et la mise en page n'est pas sans rappeler certains de ses propres calligrammes, et notamment l'un des plus réussis, «Lettre-Océan», datant de 1914. En effet, la distinction entre discours théorique et pratique poétique tend à s'effacer, comme en témoignent l'apparition de formes mixtes, de productions hybrides tels les "poèmes-manifeste» ou les «tableauxmanifeste». Cette fusion entre réflexion et poésie est représentative de l'avant-garde, selon Guillermo de Torre: "toda obra de vanguardia, en su momento más típico, fue esencialmente lírica y teórica. Poblada de versos y erizada de manifiestos» (1971: 127). Les manifestes, tout comme les proclamations et autres textes théoriques, ne doivent donc pas être mis à l'écart dans l'ensemble de l'œuvre des auteurs d'avant-garde: la philosophie de l'art est devenue pour eux une pratique poétique et les poèmes, à leur tour, constituent de véritables réflexions métalittéraires.

3 Derrière les attaques, parfois virulentes, dirigées contre l'art et la littérature antérieurs, il faut voir également une critique de l'institution artistique dans son ensemble: «El dadaísmo atacaría no sólo a las restantes corrientes - en especial, futurismo, expresionismo y cubismo -, sino a toda la institución del arte que dicta y controla los procesos de producción y recepción en el mercado cultural» (Gómez Torres, 1998: 34). 
Parmi toutes les formes textuelles de l'avant-garde, le manifeste s'est aussitôt révélé comme le modèle rhétorique dominant, à tel point qu'il était destiné à se perpétuer comme l'apport le plus emblématique de chaque mouvement. Le manifeste, d'après Ana María Gomez Torres,

aunaba lo poético y la teoría, la violencia destructora de la vanguardia y una articulación preceptiva, dogmática incluso, de las normas - o antinormas exigidas por los nuevos sistemas expresivos (1998: 12).

Le manifeste trouve sa raison d'être dans la volonté de rénovation qui caractérise les mouvements d'avant-garde: voué à l'action et toujours tourné vers le futur, il va servir de véhicule à la diffusion d'un projet artistique parfois très ambitieux qui, néanmoins, restera souvent lettre morte ${ }^{4}$. Par ailleurs, le genre du manifeste est considéré encore aujourd'hui comme l'une des contributions les plus importantes à l'histoire de la littérature et les plus originales des avant-gardes ${ }^{5}$. Bien que les manifestes littéraires ne datent pas de cette époque, c'est dans les premières décennies du $\mathrm{xx}^{\mathrm{e}}$ siècle qu'apparaît la conscience très nette d'un genre, d'un «art» spécifique et autonome ${ }^{6}$. Ce genre littéraire que l'avant-garde redécouvre et impose est, pour Adrian Marino, «la forme par excellence de l'art de la négation et du renversement» (1986: 825). Tout en faisant preuve d'un attrait marqué pour la provocation, l'humour et l'esprit ludique, le manifeste reste profondément attaché à une dynamique négative:

La rhétorique du non et de l'anti qu'on pourrait illustrer à l'infini (antitradition, antipasséisme, anticlassicisme, antiromanticisme, etc.) joue un rôle de premier plan et ce dans des textes et contextes très différents. Non et anti, vocables par excellence de l'opposition, de la révolte et de la rupture, entraînent la fréquence du mode impératif, d'un style d'ultimatum, [...]. Les manifestes expriment tous, sans exception, des décisions irrévocables, des sommations (Marino, 1986: 826-827).

4 Tel est aussi l'avis d'Ana María Gómez Torres, qui apporte plusieurs exemples dans son livre La retórica de la nada: en torno a la poética de las vanguardias: "Cuando en 1909 Marinetti publica el primer Manifiesto del Futurismo en París, aún no se había escrito una verdadera obra de esta corriente. Del mismo modo, el primer Manifiesto de la pintura futurista precedió a toda la producción pictórica del movimiento» (1998: 11).

5 «De hecho, la transmisión de los manifiestos ha llegado a alcanzar una presencia más perdurable que las obras mismas: la poesía de Marinetti, Tzara o Breton no está hoy tan viva como sus textos teóricos» (Gómez Torres, 1998: 10); "Marinetti n'a pas laissé d'œuvres littéraires plus vivantes que ses proclamations» (Weisgerber, 1986: 795).

6 Dans son premier Manifeste Dada Tristan Tzara propose une réflexion ironique sur l'«art» d'écrire des manifestes: «Para lanzar un manifiesto es necesario querer A. B. C., fulminar contra 1, 2, 3, irritarse y aguzar las alas para conquistar y propagar grandes y pequeños a, b, c, y afirmar, gritar, blasfemar, acomodar la prosa a manera de evidencia absoluta » (De Micheli, 1983: 293-294). 
Le manifeste, animé par une vocation militante qui prône l'action, par un sens critique toujours en éveil, est censé servir avant tout une finalité destructive. Par conséquent, son rituel formel impose une rigueur extrême: forme précise et concise, au service de la plus grande efficacité. Et cela, dans l'utilisation de toutes les ressources disponibles: aussi bien le langage verbal que les codes visuels et, tout particulièrement, les arts graphiques très développés au début $\mathrm{du} \mathrm{xx}^{\mathrm{e}}$ siècle, la publicité étant alors en plein essor ${ }^{7}$. Dans les avant-gardes, la pratique du manifeste se caractérise par une symbiose parfaite entre forme et message, entre technique artistique et but poursuivi, entre style et intention.

Maniluvios est l'un des livres de José-Miguel Ullán où la fusion entre théorie et pratique devient plus significative. Publié en 1972 dans la prestigieuse collection de poésie «El Bardo», il représente un tournant décisif dans son œuvre ${ }^{8}$. Ce recueil de poèmes a été conçu par l'auteur comme un ensemble unitaire dans lequel tous les composants - du contenu des poèmes aux éléments typographiques - s'intègrent dans un même projet poétique. Le titre du livre, Maniluvios, constitue d'ailleurs une synthèse parfaite de ce projet, lequel va être présenté dans le texte écrit en lettres capitales qui occupe la $4^{\mathrm{e}}$ de couverture:

LAS MANOS BORRAN LA PREHISTORIA. EL

MANILUVIO MINA LAS LÍNEAS

DE LA USANZA. RETABLO SIN

AUTOR.

(NO ANÓNIMO.)

SÓLO EL ACTOR: SUS

ADEMANES, EL AJAMIENTO DE

LA PALABRA, EL SILENCIO

LA POESÍA PRACTICA LA

DESTRUCCIÓN9.

7 «C'est dans la même ambiance que l'on met au point une foule de formes publicitaires, aussi denses qu'agressives: tracts, calicots, papillons, écriteaux, affiches, «le livre éclair», «le livre affiche» (Ilarie Voronca), dont l'efficacité est considérée comme étant à toute épreuve» (Marino, 1986: 826).

8 En fait, il est souvent considéré par la critique comme le livre qui confirme le passage d'une première étape de "poesía social » à une deuxième étape de "poesía experimental», même si certains auteurs n'approuvent pas cette division, comme Miguel Casado: «En toda la poesía de Ullán, lo existencial y lo lingüístico están fuertemente entrelazados, y los textos de intención política no son en eso distinguibles» (1994:22).

9 Dans la première édition de Maniluvios (El Bardo, 1972) la présentation visuelle du manifeste reproduit le tracé de l'écriture du poète, et il est accompagné d'une photographie avec le portrait de José-Miguel Ullán. 
D’après la définition du dictionnaire de la Real Academia de la Lengua Española, «el maniluvio» (du latin manus et luere) est « un baño de la mano, tomado por medicina, y que se utiliza también en el contexto de la liturgia religiosa». Que ce soit dans le cadre de la médecine ou de la liturgie, la finalité du maniluvio était la purification, du corps et de l'esprit. Ce manifeste, dans lequel l'écriture porte les traces de la main de l'auteur ${ }^{10}$, précise la signification du maniluvio dans un contexte littéraire, en reprenant deux notions essentielles de la poétique des avant-gardes: le désir de renouveau et l'importance accordée à l'action.

Le recours à la rhétorique de la négation, caractéristique de l'art du manifeste, est mis en évidence par la violence de l'acte accompli: les verbes «borrar», «minar» et les substantifs «el ajamiento», «la destrucción». La présence de ce dernier mot permet d'établir une relation intertextuelle avec les avant-gardes historiques, puisqu'il renvoie au titre-programme d'œuvres comme Destruction (1904) de F. T. Marinetti, Distruzione (1911), de Luigi Capuana ou Décomposition (1918), de Tristan Tzara. La filiation intertextuelle pourrait même remonter jusqu'au poète Stéphane Mallarmé qui, dans Propos sur la poésie, confie: «La Destruction fut ma Béatrice» (1953: 91). La notion de «destruction» est fondamentale dans les avantgardes, dans la mesure où, pour pouvoir construire du «nouveau», il faut d'abord détruire l'«ancien», repartir à zéro. La négativité pousse à l'anéantissement, but de la révolte: "Il faut renverser avant de restaurer" (Weisgerber, 1986: 777). Mais de cette manière, la voie est ouverte à des créations nouvelles, à des expressions d'aspirations positives ${ }^{11}$. La polarité entre destruction et construction est omniprésente, et pour preuve, la

10 En plus de l'importance accordée à la dimension visuelle et de l'originalité expressive qu'implique cette particularité graphique, le choix de l'écriture manuscrite permet également d'établir un lien avec les avant-gardes: "La lettre ne se résigne plus à n'être qu'un simple signe. Elle se charge de personnalité, se transforme (aux yeux des futuristes russes d'abord) en "composante de l'impulsion poétique». Il y a une très grande différence entre l'écriture de l'auteur, celle du copiste et les caractères d'imprimerie: la présentation la plus poétique sera autographe - le livre que l'auteur écrit «pour soi» (Weisgerber, 1986: 37).

11 «On aurait pourtant tort d'identifier intégralement l'esprit, le programme et l'art de l'avant-garde à une négation perpétuelle, à une entreprise de démolition stérile. En vérité, la dialectique ancien/nouveau, qui régit ces mouvements dans leur ensemble, est à la fois négative et positive, destructrice et constructrice. C'est là une dualité fondamentale: l'avant-garde ne démolit la tradition qu'afin d'instaurer sur ses décombres un ordre nouveau. S'il est parfaitement vrai que sa nouveauté consiste au départ à s'insurger, il n'empêche que cette détermination négative a pour cause première et pour principe directeur une puissante volonté d'affirmation» (Weisgerber, 1986: 703). 
division en deux parties ( «DESTRUCTION $/$ / «CONSTRUCTION») du manifeste l'Antitradition futuriste de Guillaume Apollinaire. Pour les avant-gardes, l'action destructrice implique tout d'abord le renversement de l'ordre établi, l'anéantissement de l'héritage transmis par la tradition, étape nécessaire à toute création vraiment nouvelle. L'avant-garde se définit comme une théorie et une pratique radicalement antitraditionnelles:

Le passé, source de tous les principes, hiérarchies et valeurs traditionnels, est bien la bête noire des avant-gardes. L'antitradition est la clef de voûte de tous leurs manifestes (Weisgerber, 1986: 633).

De même, dans le manifeste manuscrit de Maniluvios, le désir de renouveau est indissociable de la volonté destructrice: "practica la destrucción». La volonté de rénovation entraîne le rejet du passé, «borran la prehistoria» et le refus des conventions, «mina las líneas de la usanza».

La déclaration finale annonce la mission de l'écriture poétique: «la poesía practica la destrucción » et son objet: «el ajamiento de la palabra, el silencio». Dans sa formulation comme dans son dessein, cette déclaration rejoint un projet partagé par la plupart des avant-gardes, pour qui la destruction s'exerce, en premier lieu, sur le langage: désarticuler l'écriture, s'attaquer aux mots, "déconstruire le bel édifice linguistico-philosophicolittéraire» (Weisgerber, 1986: 678). Des avant-gardes aux néo avant-gardes, presque tous les mouvements participent de la même tendance, devenue une constante théorique: ébranler les fondements du langage dans l'intention de contester, par la même occasion, les principes de base de la société.

C'est dans les milieux d'avant-garde où les rêves d'une révolution sociale, politique et littéraire se côtoient et parfois se confondent que l'aspiration vers la révolution du langage devient radicale (Weisgerber, 1986: 651).

À travers le double versant de cette critique, les mouvements d'avant-garde aspirent à un renversement aussi bien dans le domaine de la langue que dans l'univers social: "Pour saper le régime dominant, il faut s'attaquer simultanément au langage qui l'exprime et, en quelque sorte, le cautionne» (Weisgerber, 1986: 651). De cette manière, pour les avant-gardes, la transformation du monde passe inévitablement par la régénération du langage ${ }^{12}$.

12 Pour Weisgerber, la dimension sociale et politique est essentielle: "Au-delà de ces formules, c'est l'ordre établi de la culture tout entière qui est visé dans ses assises. L'ennemi à abattre est le système dominant pris dans son ensemble, élevé au rang d'héritage culturel, avec son appareil, sa discipline, sa hiérarchie» (1986: 634). 
«Las manos borran la prehistoria». Dans le manifeste manuscrit, l'acte de destruction est accompli par «las manos». Fragmentation du sujet, dont la participation est limitée à la seule présence des mains, mettant ainsi en évidence la prééminence donnée au geste, à l'action: "sus ademanes». Le sujet lyrique s'efface, cédant sa place à cette instance impersonnelle que constitue le maniluvio: "El maniluvio mina las líneas de la usanza». Le manifeste revendique la disparition de l'auteur: «retablo sin autor», remplacé par l'acteur: «sólo el actor». La notion d'auteur - conçu en tant que responsable individuel du texte - a été particulièrement mise à mal par les avant-gardes, qui multiplièrent les expériences où la conception traditionnelle de l'auteur était sérieusement remise en cause.

Con el ready-made se despedía la noción de «obra maestra» y se agredía el concepto de creación individual; Dadá practicó el arte colectivo; los manifiestos del futurismo ruso eran firmados por varios autores, al igual que algunas de las proclamas ultraístas; el autor se hacía casi anónimo en los actos colectivos de las vanguardias; los poemas simultáneos eran recitados por varias voces en las veladas Dadá, y el collage poético integró una pluralidad de fragmentos y citas de otros autores, en un juego dialógico de intertextualidades (Gómez Torres, 1998: 38).

Pour s'en prendre à la notion traditionnelle d'auteur, plusieurs procédés sont mis en œuvre : que ce soit grâce à des productions plurielles ou anonymes, tels les travaux collectifs concernant la création de l'œuvre littéraire, ou bien le postulat du hasard créateur. Mais également des techniques comme le ready-made ou encore, en rendant effective la disparition de l'auteur en tant qu'origine de l'acte de production, par exemple lorsque la voix de l'écrivain est étouffée par des bruits jusqu'à la rendre inaudible, inexistante ${ }^{13}$. En réalité, cette attaque fait partie d'une offensive plus globale, l'anticréation, où aucun représentant de l'institution artistique ne fut épargné14. Comme l’affirme Ortega y Gasset dans son œuvre La deshumanización del arte, «al

13 «Dada cultivó un antiarte de lo efímero: en las veladas parisinas de 1920, Picabia ejecutaba sus dibujos sobre una pizarra, para borrarlos a continuación; cuando Tzara leía un poema, su voz era apagada por el sonido de un timbre eléctrico» (Gómez Torres, 1998: 35).

14 «On commence par ruiner d'un seul coup la notion de «création» en tant que productrice d' "œuvres d'art». Et cela de deux manières. D'abord par une opération de dépersonnalisation, d'effacement voulu du créateur individuel, de son identité en tant que nom et agent. Le poème simultané, conçu et récité spontanément par plusieurs auteurs, correspond à cette première méthode. Ensuite, par le remplacement de l' «œuvre faite» (portant une signature, etc.) par des objets trouvés, les fameux ready-mades de Marcel Duchamp, dont la suprême qualité est de se situer totalement en dehors du monde de l'art» (Weisgerber, 1986: 687). 
fin y al cabo, agredir al arte del pasado es revolverse contra el Arte mismo " (1964: 56). En remettant en cause la tradition artistique, en critiquant les institutions, les avant-gardes s'insurgent contre le statut de l'art dans la société.

Dans son manifeste manuscrit, José-Miguel Ullán oppose «el autor» à «el actor». Il ne s'agit donc pas de la disparition totale de l'auteur, mais plutôt de sa substitution par l'acteur. En effet, l'auteur continue, d'une certaine manière, à être présent, comme le texte lui-même l'indique: «no anónimo", ou comme le suggère le tracé de l'écriture, réalisé par le poète de sa propre main. Le glissement de l'auteur vers l'acteur fait apparaître la confrontation entre deux conceptions opposées de la littérature. L' «autor» correspond à la notion traditionnelle, celle de l'institution littéraire, tandis que l'«actor» appartient à une nouvelle vision de l'écriture poétique. Le manifeste manuscrit partage avec les avant-gardes le rejet de la définition de la poésie héritée du romantisme: «Certains aspects, et non des moindres, de la tradition romantique sont également rejetés» (Weisgerber, 1986: 646), du poème exclusivement articulé autour du sujet:

Il s'agit, bien sûr, d'une opposition au romantisme conventionnel, mais aussi d'un antiromantisme à programme, qui vise en profondeur le lyrisme, la subjectivité, la littérature-confession et la littérature sentimentale (Weisgerber, 1986: 646).

Par ailleurs, José-Miguel Ullán a exprimé à plusieurs reprises son refus d'une conception lyrique qui perçoit le poème comme l'expression du ressenti du «moi poétique», du vécu du «je» de l'auteur. Et surtout lorsque ce recours à des éléments biographiques risque d'entraîner - même dans l'exercice de la critique littéraire - la confusion entre biographie et poésie ${ }^{15}$. De plus, la mise en question de l'auteur témoigne de la crise du sujet qui caractérise la plupart des manifestations artistiques et littéraires dans le premier tiers $\mathrm{du} \mathrm{xx}^{\mathrm{e}}$ siècle. Cette critique de la notion d'auteur va être reprise par les néo avant-gardes, tout comme le thème de la «mort de l'auteur», essentiel dans le contexte intellectuel des années 60-70 et, entre autres, dans Roland Barthes ou Michel Foucault.

15 Dans l'entretien avec Ramón Chao publié à l'occasion de la parution de Maniluvios, José-Miguel Ullán tient à déclarer: " Nuestros paisanos, para camuflar su ignorancia ante la realidad de la escritura, demuestran un apetito desenfrenado por el chismorreo biográfico: única y lamentable base sobre la que se alzan sus glosas críticas y comentarios epidérmicos» (Chao, 1972: 45). 
Les parallélismes entre le texte de José-Miguel Ullán et le genre du manifeste, tel qu'il fut conçu et développé par les avant-gardes, sont nombreux : la présence de la rhétorique de la négation, le recours à la violence verbale, ainsi que le choix d'un style saccadé qui relève de l'injonction, du mode impératif. La cohérence entre le fond et la forme est mise au service de la plus grande efficacité, et une attention toute particulière est accordée à la composition visuelle du texte: la disposition des blancs et des mots sur la page joue un rôle primordial dans la transmission du contenu métalittéraire. Il paraît donc évident que José-Miguel Ullán a eu recours au genre du manifeste, mais pour quoi faire? En tant que modèle textuel extrêmement productif, l'efficacité du message semble, bien sûr, assurée. Et, en tout état de cause, il s'agit d'une référence intertextuelle que le lecteur doit être en mesure d'identifier, étant donné qu'elle est essentielle à la création du sens dans le manifeste manuscrit, et même, à la signification du livre en tant que projet poétique. En outre, en faisant appel à ce cadre générique, le poète choisit un moyen de revendiquer l'héritage des avant-gardes, de se présenter comme faisant partie d'une "tradition de la rupture» dont les origines remontent aux dernières décennies du $\mathrm{XIX}^{\mathrm{e}}$ siècle. Néanmoins, la distance qui sépare son œuvre des avant-gardes historiques est, bien évidemment, considérable. José-Miguel Ullán ne s'érige pas en porte-parole d'une mouvance collective, il n'a aucune prétention de fonder un mouvement quelconque, ou de s'opposer à un autre mouvement préexistant: son projet artistique relève d'une démarche strictement individuelle, sans aspirer non plus à atteindre une portée plus générale, comme c'est le cas dans les avantgardes. Cependant, l'essentiel du genre du manifeste en tant que réflexion théorique mais aussi en tant que pratique textuelle est maintenu, le but à atteindre restant toujours le même: le refus des conventions et des normes consacrées par l'institution, la défense à outrance d'une poétique nouvelle.

\section{De la poésie à la poétique: la réflexion métalittéraire}

L'art du $\mathrm{xx}^{\mathrm{e}}$ siècle se caractérise, pour Umberto Eco, par la prééminence de la poétique sur la poésie, de l'idée ou projet théorique sur le produit final: «el problema de la poética ha prevalecido sobre el problema de la obra en cuanto cosa realizada y concreta (1985:256). Dans les avant-gardes, l'œuvre de création se présente comme un manifeste poétique et philosophique sur la représentation artistique, sur la vision du monde dans l'art. Pour sa part, le manifeste, dispositif rhétorique et donc écrit en prose, fait appel à des pratiques qui appartiennent à l'écriture poétique. Dans Maniluvios, le texte 
manuscrit, de nature théorique, emprunte au poème sa composition visuelle, en créant des effets de sens - la fonction des "blancs», la "suspension" provoquée par l'enjambement - spécifiques à la poésie. Quant aux poèmes, la singulière distribution des vers sur la page va les situer dans la limite entre prose et poésie, entre la disposition linéaire et continue de la phrase et le découpage imposé par le schéma rythmique de la versification.

[LOS Limites DEL POEMA]

todo es azar el papel

y la herida que lo habi

ta mas necesita eso sí

un raro candil -la sed

La disposition visuelle du poème est délibérément trompeuse: le «bloc» de texte "[LOS LIMITES DEL POEMA]» se compose en réalité de quatre vers octosyllabes. Dans un jeu cher à José-Miguel Ullán - les exemples sont nombreux dans son ouvre -, le poème masque sa nature poétique sous l'apparence de la prose. Miguel Casado décrit ainsi cette particularité, présente également dans d'autres formes textuelles de Maniluvios: «esas cajas de prosa son recorridas por armonías en que se reconoce la huella del verso clásico : la prosajuega a recortarse como verso y el verso fluye en la continuidad de la prosa» (1994: 46). La mise en page du poème crée une dissonance entre l'aspect visuel et le modèle métrique, entre la musicalité des vers et leur agencement dans l'espace. Face à l'absence de ponctuation, le blanc, ou encore, les signes typographiques comme le tiret, ont une fonction structurante, en plus de constituer des points de repère dans le tracé d'un parcours de lecture à l'intention du lecteur.

La pertinence d'une lecture métalittéraire du poème est confirmée aussi bien par son titre, "[LOS LIMITES DEL POEMA] » que par ses premiers mots: «todo es azar» renvoie à Mallarmé et à son célèbre Un coup de dés jamais n'abolira le hasard. En fait, tous les substantifs du poème peuvent être interprétés du point de vue du discours métapoétique. Si «el papel» constitue une image de la page écrite et par extension du poème, pour comprendre la signification de «el candil», «la sed», et «la herida» il faut se tourner vers le premier livre de l'auteur, El Jornal, publié en 1965. Un poème-poétique inaugure le recueil de poèmes: "Amatando el candil/ tan en mi hogar.// Y, sin embargo, cósmico». Trois mots, trois images pour désigner le désir, la lumière éclatante dans l'obscurité, la «voluntad de riesgo » qui se trouvent à l’origine de l'expérience poétique de José-Miguel Ullán. 
Le poème doit donc être lu comme une poétique: «todo es azar el papel / y la herida que lo habi/ ta». La suprématie accordée au hasard dans le processus de création poétique revient à confirmer la disparition de l'auteur, revendiquée dans le manifeste manuscrit. Et en effet, "[LOS LIMITES DEL POEMA] » ne laisse deviner aucune trace de la voix de l'auteur ou du «je» poétique: il se présente au lecteur comme un objet autonome, entièrement bâti autour de la réflexion métalittéraire, son seul contenu référentiel. Ce poème est par ailleurs le dernier d'une série dont la dimension métalittéraire est très clairement mise en évidence dans les titres: "[EL UMBRAL DEL POEMA]», "[LA RESISTENCIA DEL POEMA]», "[NACIMIENTO DEL POEMA]». La progression de la série s'identifie au cycle vital d'un être animé, dont la naissance s'assimile au processus de création littéraire. De plus, cette progression renforce le caractère autonome des poèmes, en suggérant qu'ils «naissent», qu'ils se créent à l'instant même où le regard du lecteur se pose sur la page, et cela de manière indépendante, sans la participation de l'auteur ou de tout autre intervenant extérieur au texte. D'après Weisgeber, l'avantgarde conçoit le processus de création poétique comme autonome: "Une fois le processus déclenché, il ne fait que se soumettre à ses normes internes» et en outre, l'autonomie en tant que notion «souligne la conviction que la création suit ses propres lois, se développe selon sa propre structure et la spécificité de ses moyens d'expression» (1986: 757). De même, la conception du poème comme un objet autonome est indissociable de la vocation métalittéraire de la poésie moderne, de Mallarmé à Reverdy, et confirme l'hégémonie de la poétique sur le contenu référentiel, de l'idée ou projet théorique sur la production artistique.

Dans Maniluvios, la fusion entre discours théorique et pratique poétique s'accomplit également dans les poèmes, lesquels deviennent le véhicule d'expression d'un message métalittéraire, d'une réflexion sur la poésie. Le projet poétique exposé dans le manifeste manuscrit se réalise dans un modèle textuel très singulier: la caja de prosa, que José-Miguel Ullán explore dans la série de six poèmes appartenant à la deuxième partie du livre, intitulée «[EL POEMA] ». Le premier texte de la série, «1», est particulièrement significatif en ce qui concerne ces pratiques textuelles innovantes dont le contenu est surtout métalittéraire. 
1

inteligencia no me dés jamás el nombre exacto de las cosas $\mathrm{p}$ orque el enlabio se enniñece oh sí y los molinos del aliento casi borran orillas al volver los copos se enciende un sordo crepitar de arrugas tibias y leves porque el sol se vela gui ña se alueña como moro a pasas y las pestañas se entrecruza $\mathrm{n}$ hierve la alegre espiga vespertina inútil tal toda dicha $\mathrm{d}$ e repente ahora que ya en el ring tan sólo flota un chorro d e adondequiera o duérmete mas vuelan blandas estrellas chamuscados pétalos remos de fiebre por las aguas suches del mar sin norte ya en pelotas abren el arca malva de la he rencia sajan las vagas señas invernales bocas que se bendice n con albor miradas libres de espejo por las uñas una lúa qu e aclara otra corteza errante pausa de luz de su vejez más $\mathrm{p}$ róxima y bueno y qué genuflexión la saña el tole tole del te rror ahueca piel y saliva cabrilleas limo picha solemne som bra cana $o$

las leyes finan

dura el borrajo y la mar aña intacta de una caricia inacabada entones mu mu es la muerte la vigilia el gayo insecto en torno al agujero oh sí

L'appellation caja de prosa fait référence à une disposition particulière du texte poétique dans l'espace: un «bloc» aux contours parfaitement bien définis, mais avec un degré d'ouverture variable à l'intérieur. Le mot caja appartient au champ lexical de l'imprimerie: la «caja» correspond, d'après la définition du Diccionario de la Real Academia de la Lengua Española, à «el espacio de la página lleno por la composición impresa». La caja de prosa renvoie donc à une singulière mise en page des mots, dans laquelle les bords du texte écrit sont tracés conformément à un dessin rectangulaire imposé, en respectant un encadrement établi à l'avance. La caja de prosa représente l'image textuelle de "la caja de imprenta», du cadre qui découpe les lettres en forme de carré sur la page blanche. De ce fait, cette forme textuelle devient aussi une métaphore de l'acte d'écriture lui-même.

Cette disposition visuelle n'est pas sans conséquence quant à la structure interne du texte. La forme visuelle l'emporte sur le respect de l'unité des phrases, de la cohérence syntaxique ou logique. Comme l'a décrit Miguel Casado, dans la caja de prosa:

se produce un choque entre la palabra escrita y la página, o mejor, la mancha tipográfica: el cuerpo del discurso escrito se encierra en un tácito y estrecho 
dibujo rectangular que no respeta la palabra. El conflicto se resuelve a favor del diseño de la página y las reglas de división silábica saltan hechas trizas; cada línea tiene la misma medida total y la palabra se corta en cualquier sitio, allí donde la línea acaba (1994: 45).

Pour Casado, la coupure arbitraire subie par les phrases du texte rappelle le «moule» métrique imposé par la versification, et il propose une interprétation de ce mécanisme imprévisible comme «una irónica readaptación de los principios que explican la rima o el isosilabismo» (1994: 46). La caja de prosa, à nouveau, se situe dans la limite entre prose et poésie, et cela d'autant plus qu'il est possible d'y reconnaître des rimes internes, des assonances et des allitérations. Cependant, la création de ces effets poétiques échappe à la volonté de l'auteur, puisqu'elle est confiée à un mécanisme aussi implacable qu'arbitraire. En effet, c'est la disposition visuelle imposée qui coupe les mots au hasard, en créant des associations visuelles et sonores inattendues, des jeux de mots, des effets de surprise... Cette confiance dans les capacités créatrices du hasard («todo es azar») est liée à la remise en question des principes de la création littéraire et artistique traditionnelle. Comme dans les avant-gardes, la revendication du rôle du hasard est associée à une critique dont le sens polémique, très précis, se dirige principalement contre l'acte créateur ${ }^{16}$.

Le poème « 1 » est également porteur d'un message métalittéraire qui met en évidence son propre principe de création. En fait, la disposition des blancs permet de mettre en valeur une phrase particulièrement significative: «las leyes finan». D’après Weisgerber, la fonction de l'avant-garde «consiste à s'élever périodiquement contre l'instauration de toute norme" (1986: 657). L'art, la littérature, la poésie ne connaissent pas de «lois». Cette revendication représente une des principales consignes des mouvements d'avant-garde: "La révolte contre la tradition aboutit à une contestation et à un éclatement du langage en tant que produit de normes, thèmes et formes

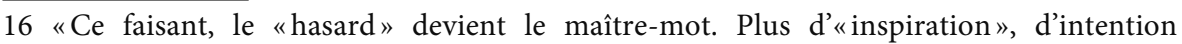
créatrice, d'esquisses préparatoires, de plans. Le hasard devient la source même de la création: le verre cassé de Marcel Duchamp, ou les mots découpés d'un article, fourrés dans un sac d'où l'on sort chaque coupure l'une après l'autre, pour les recopier consciencieusement au fur et à mesure. C'est la recette - célèbre - intitulée Pour faire un poème dadaïste (1920) et qui compte certains précurseurs, tout au moins théoriques. Après Dada, l'ultraísmo veut qu'on écrive selon le même procédé («arrojando las palabras al azar»). Le surréalisme s'adonnera à une observation extrêmement attentive, presque obsessionnelle, des coïncidences et des autres phénomènes dits de "hasard" (Weisgerber, 1986: 688). Dans le contexte des néo avant-gardes, cette confiance dans le hasard comme mécanisme de production rappelle bien sûr les productions du groupe Oulipo. 
périmés» (1986: 656). Le contenu métapoétique de «1» s'accorde avec le manifeste manuscrit: nier simultanément l'idée traditionnelle de création, de littérature ou d'art, ainsi que l'ensemble des valeurs héritées du passé.

La réflexion métalittéraire exprimée dans "[LIMITES DEL POEMA]» et « 1 » tient le poème pour un univers autonome, aux lois arbitraires, fruit du hasard. La poétique de la caja de prosa est en parfaite cohérence avec le projet artistique exposé dans le manifeste de Maniluvios: disparition de l'auteur, suprématie accordée au hasard, refus des normes et des conventions traditionnelles, destruction du langage.

\section{De la littérature aux arts plastiques: la caja de prosa}

Dans la littérature d'avant-garde, les catégories génériques s'effacent, la théorie n'est plus séparée de la pratique, le manifeste et le poème se confondent dans une production unique. De même, la distinction entre prose et vers est abolie:

la prose se poétise, la poésie tourne à la prose. D’où la fréquence du texte, œuvre inclassable au sens où fait défaut le cadre ou patron consacré par l'usage (Weisgerber, 1986: 796).

La caja de prosa partage des caractéristiques des deux genres: l'image de l'écriture sur la page correspond bien à la prose, mais le tissu rythmique, les rimes et les assonances sont spécifiques à la poésie. Par ailleurs, l'importance accordée à la disposition visuelle du poème sur la page rapproche le modèle textuel de la caja de prosa des arts plastiques: la dimension graphique du mot est aussi importante pour la création du sens que son contenu sémantique.

La disposition visuelle de la caja de prosa s'inspire d'un modèle textuel mis en œuvre dans les avant-gardes historiques et repris ensuite par les néo avant-gardes. Dans ses Sept manifestes dada, Tristan Tzara conçoit un «bloc» bâti sur onze colonnes avec vingt-cinq mots chacune, où se répète inlassablement un seul verbe: "hurle $»^{17}$. Le texte a une nature «mixte»: s'il fait partie des Sept manifestes, sa forme textuelle correspond à la poésie visuelle ou concrète, telle qu'elle fut pratiquée par les avant-gardes historiques et ultérieurement par les néo avant-gardes. La répétition est un

17 Il s'agit du texte $\mathrm{n}^{\circ}$ XVI, signé par l'auteur lui-même, et inclus dans Euvres complètes. Tome I (1912-1914), (1975: 387). Je tiens à remercier Túa Blesa de m’avoir proposé de faire le rapprochement entre la caja de prosa et le texte de Tristan Tzara. Pour l'étude d'autres formes textuelles novatrices dans la poésie espagnole contemporaine, il faut consulter son livre Logofagias. Los trazos del silencio, Zaragoza, Anexos de Tropelías, 1998. 
procédé courant dans les manifestes, dans un but d'efficacité. La nouveauté réside ici dans la disposition visuelle, qui s'adapte parfaitement à la finalité poursuivie : la régularité a un effet d'insistance. Telle une caisse de résonance, l'amplification du volume, de l'intensité du son prononcé, résultent du procédé visuel de la répétition du même mot. En parfaite cohérence avec l'esprit subversif et la violence verbale propres aux mouvements d'avantgarde, la quête d'une nouvelle expressivité contribue aussi à renfoncer l'efficacité du message. Comme l'affirme Ricardo Senabre:

el tratamiento vanguardista de los textoshace recaer la atención sobre la propia forma del mensaje, sea por su disposición gráfica, sea por el modo insólito, nuevo y sorprendente, de presentar un hecho consabido y familiar (1999: 211).

Le recours aux codes spécifiques des arts visuels dans la création littéraire a également comme but de bousculer les mécanismes de réception habituels. En cherchant à franchir ses propres limites, la littérature se rapproche de l'art, la peinture de la poésie, dans un même élan qui les porte vers l'aspiration à un " $\operatorname{art} » \operatorname{total}^{18}$.

Dans les néo avant-gardes, la pratique de la poésie concrète ou visuelle prend une ampleur considérable, et les convergences entre les expériences de l'art plastique et la littérature se font de plus en plus nombreuses. Par ailleurs, l'héritage des avant-gardes historiques reste bien vivant, comme en témoigne le texte "und zer brechen" de Gerhard Rühm, inclus dans l'anthologie Concrete poetry: a world view (Solt, $1971: 133$ ). Ce poème est construit sur le même procédé de répétition que «XVI» de Tristan Tzara. L'expression « und zer brechen » pourrait se traduire en français par «et se brise». À différence du modèle de son prédécesseur, le mot «und» est attaché tout au long du texte, pour mieux souligner la rupture qui survient lorsque la phrase «un zer brechen» apparaît en entier à la fin. La composition graphique devient hautement significative, puisque les mots représentent visuellement leur

18 Ces particularités sont bien le résultat de l'abolition des frontières entre les genres et entre les arts, d'après Michel Dupuis: "On vient de voir, à plusieurs reprises, les catégories génériques s'effacer au sein de la littérature d'avant-garde, et celle-ci se confondre, au-delà, avec les autres modes de création artistique. Faut-il rappeler, encore une fois, que bien des manifestes abolissent toute distinction entre théorie et pratique, entre exposé et poésie; qu'un poème concret se « regarde», tout comme doit se «lire» telle toile de Magritte; ou qu'un spectacle de pointe «se vit»? L'avant-garde trahit bien par là sa volonté d'agir sur tous les centres nerveux du public, de tirer parti de toutes les facultés à la fois, d'étendre sa force de frappe en même temps qu'elle l'intensifie. L'œuvre «totale» dont elle rêve ne s'adresse plus (ou plus uniquement) au seul bon sens, à la seule logique, à la perception banale des choses» (1984: 877). 
propre contenu sémantique. Cependant, l'attention accordée à la disposition visuelle des lettres et des mots ne doit pas être confondue avec un simple formalisme ou jeu typographique: la finalité ultime du rapprochement avec les arts plastiques est la volonté de multiplier les possibilités expressives, la capacité à créer des nouvelles significations.

Dans la poésie d'avant-garde, le poème est conçu comme un objet autonome, qui demande au lecteur une double compétence: il demande à être vu en même temps que lu. La lecture du poème implique nécessairement le regard attentif de celui qui contemple un tableau. Depuis Les mots en liberté futuristes de Marinetti, et même avant, avec Mallarmé et Un coup de dés jamais n'abolira le hasard, la typographie va rythmer la presque totalité des poèmes d'avant-garde et les situer dans un nouvel espace graphique. La disjonction des éléments provoquée par l'invasion de l'espace est responsable de la dispersion syntaxique. Le poème ne constitue plus un ensemble dont la syntaxe assure la cohésion rationnelle, la rupture de la linéarité ayant supprimé le processus causal de la discursivité: "Non seulement le poème s'œuvre sur l'espace, mais il constitue, grâce à lui et à travers lui, une autonomie qui refuse toute autre référence que celle même de sa propre origine» (Verhesen, 1984: 802). Pour la poésie d'avant-garde, la disjonction typographique fut un moyen de briser le sens discursif, de se libérer des contraintes rationnelles. Elle s'attaque à l'univocité du langage et entraîne la dispersion des signifiants, qui apparaissent davantage comme des traces que comme des signes référentiels: «le mot en considéré comme contenant, en sa forme pure, des sens multiples que le récepteur-lecteur est invité à pénétrer, à déployer à son gré» (Verhesen, 1984: 802). La complicité du lecteur devient essentielle, puisqu'il est invité à parcourir les multiples chemins de lecture que lui offre le texte, et à participer activement à la création du sens.

Quelle peut bien être la signification du recours à ce modèle textuel par José-Miguel Ullán? Étant donné que les ressemblances, du point de vue visuel, sont évidentes, il ne peut s'agir que d'un clin d'œil volontaire, d'une référence intertextuelle que le lecteur est censé pouvoir reconnaître. En plus de sa particulière disposition visuelle, les poèmes de Maniluvios possèdent également une dimension théorique et par conséquent, ne sont pas très loin du caractère réflexif du manifeste. Comme les textes de Tzara ou Ruhm, ils présentent une nature "mixte», à mi-chemin entre le manifeste et le poème. Par ailleurs, la caja de prosa, en plus d'être porteuse d'un message métalittéraire constitue, en ce qui concerne les aspects formels, linguistiques 
et visuels, une parfaite illustration - une application "pratique» - des principes théoriques. Grâce à cette disposition visuelle particulière, qui met en évidence la disparition de l'auteur et le refus des conventions, qui révèle le poème comme un objet autonome soumis aux mécanismes du hasard, la caja de prosa réalise le projet poétique exposé dans le manifeste manuscrit de Maniluvios: «la poesía practica la destrucción».

En conclusion, le recours à des pratiques empruntées aux avantgardes permet à l'auteur d'aborder dans son œuvre le questionnement des conventions d'écriture et de lecture, ainsi que l'abolition des frontières entre le vers et la prose, entre théorie et poésie, entre la littérature et les arts. Ces références intertextuelles et interartistiques s'inscrivent dans une démarche de réflexion sur la création littéraire, sur la représentation artistique, et enrichies d'un regard nouveau, elles sont mises au service de la poétique de José-Miguel Ullán: explorer la relation problématique entre le langage et le monde.

\section{Bibliographie}

Blesa, T., 1998, Logofagias. Los trazos del silencio, Zaragoza, Anexos de Tropelías.

Casado, M., 1994, « Introducción », Ardicia. (Antología poética, 1964-1994), Madrid, Cátedra Letras Hispánicas, p. 11-150.

Chao, R., 1972, «José-Miguel Ullán o la destrucción », Triunfo n 517: 44-45.

Eco, U., 1985, La definición del arte, Barcelona, Planeta.

De Micheli, M., 1983, Las vanguardias artísticas del siglo xx, Madrid, Alianza.

Dupuis, M., 1986, "L'œuvre d'avant-garde», in: Les avant-gardes littéraires au XX siècle: Théorie, vol. II, Akadémiai Kiadó, Budapest, p. 877-881.

Gómez Torres, A.M., 1998, La retórica de la nada: en torno a la poética de las vanguardias, Málaga, Publicaciones del Congreso de Literatura Española Contemporánea.

Mallarmé, S., 1953, Propos sur la poésie, Paris, Ed. Henri Mondor.

Marino, A., 1986, "Le manifeste», in: Les avant-gardes littéraires au $\mathrm{XX}^{e}$ siècle: Théorie, vol. II, Akadémiai Kiadó, Budapest, p. 825-834.

Ortega y Gasset, J., 1964, La deshumanización del arte, Madrid, Revista de Occidente.

Senabre, R. , 1999, Claves de la poesía contemporánea. De Bécquer a Brines, Salamanca, Almar.

Solt M. E., 1971, Concrete poetry: a world view, Bloomington, Indiana University Press. 
Torre, G. de., 1971, Historia de las literaturas de vanguardia, vol. I, Madrid, Guadarrama.

Tzara, T., 1975, Euvres complètes. Tome I, (1912-1924), Paris, Flammarion.

Ullán, J.-M., 1965, El Jornal, Salamanca, Vitor.

Ullán, J.-M., 1972, Maniluvios, Barcelona, El Bardo.

Ullán, J.-M., 1972-1985, Funeral mal, RLD, París.

Ullán, J.-M., 1994, Ardicia. (Antología poética, 1964-1994), Madrid, Cátedra.

Ullán, J.-M., 2008, Ondulaciones. Poesía reunida (1968-2007), Madrid, Galaxia Gutemberg.

Verhesen, F., 1986, «La poésie», in: Les avant-gardes littéraires au XX ${ }^{e}$ siècle: Théorie, vol II, Akadémiai Kiadó, Budapest, p. 798-823.

Weisgerber, J., (sous la direction de), 1986, Les avant-gardes littéraires au $\mathrm{XX}^{e}$ siècle: Histoire, t. 1., Théorie, t. 2., Akadémiai Kiadó, Budapest. 